\title{
Production and Reaction of Singlet Oxygen in Aqueous Micellar Solutions Using Pyrene as Photosensitizer
}

\author{
Norio Miyoshi and Giiti Tomita \\ Institute of Biophysics, Faculty of Agriculture, Kyushu University, Fukuoka 812, Japan
}

Z. Naturforsch. 33b, 622-627 (1978); received February 24, 1978

Singlet Oxygen, Pyrene, Diphenylisobenzofuran, Micelles

\begin{abstract}
The reaction of singlet oxygen with 1,3-diphenylisobenzofuran was investigated in aqueous micellar solutions of sodium dodecyl sulfate and dodecyl trimethylammonium chloride using pyrene as photosensitizer. Singlet oxygen, produced by the photosensitiza. tion of pyrene bound to micelles, oxidized efficiently 1,3-diphenylisobenzofuran bound to micelles. The quantum yield for singlet oxygen production and the rate constant for furan oxidation were determined by the kinetic analysis for the oxidation reaction of furan. Quenching of singlet oxygen by sodium azide competed with the furan oxidation, and its rate constant was also determined.

Results obtained were compared with those in methanolic solutions. The quantum yield for singlet oxygen production and the rate constant for furan oxidation were much higher in micellar solutions than those in methanolic solutions. Empty micelles was found to act as a considerably strong scavenger for singlet oxygen. The penetration probability of singlet oxygen from aqueous phase into the interior of micelles which bound furan was also estimated.
\end{abstract}

\section{Introduction}

Singlet oxygen [1-5] has attracted a great attention of various workers as a reactive species from photochemical and photobiological importance. This molecule can be produced photochemically by direct excitation $[1,2,6]$ of oxygen with laser or by energy transfer [1-4] from triplet state of sensitizer to oxygen. Various dyes and aromatic hydrocarbons such as rose bengal [7-9], eosine $\mathrm{Y}[10]$, thionine $[9,10]$, methylene blue $[9,10,12,14]$ and pyrene [15-21] are known as photosensitizer for singlet oxygen production. Among these, methylene blue is most popular as sensitizer, and has been widely employed for investigating the lifetime $[7,8]$ of singlet oxygen and the photooxidation reactions of many organic substrates in various solvents [12]. Usually, dye-sensitized photooxidations proceed with singlet oxygen mechanism (type II) and/or with radical mechanism (type I) involving the reaction of excited state of dye with substrate. Sodium azide $[9,17,18]$, DF $[6-8,12], 9,10$-di-

Abbreviations: SDS = sodium dodecyl sulfate; DTAC = dodecyl trimethylammonium chloride; $\mathrm{P}_{\mathrm{y}}=$ pyrene; $\mathrm{DF}=1,3$-diphenylisobenzofuran; $\mathrm{MeOH}=$ methanol.

Requests for reprints should be sent to Prof. G. Tomita, Institute of Biophysics, Faculty of Agriculture, Kyushu University, Fukuoka 812, Japan. methylanthracene [6, 7], tetramethylethylene [7], tetraphenylcyclopentadienone [6], carotenoids [12] and amines $[17,18]$ are known as effective quenchers for singlet oxygen. Reactions concerning with singlet oxygen have been investigated using the above quenchers; especially, sodium azide in polar solvents and DF in non-polar sni- nts.

Recently, photochemical reactions sensitized by methylene blue were kinetically studied with aqueous SDS micellar systems [21] containing DF, and the behaviour of singlet oxygen was discussed. Micellar solutions provide a hydrophobic environment (interior of micelles) in polar phase. Methylene blue may be bound to the surface of micelle and $\mathrm{DF}$ to the interior of micelle. Such micellar systems are of interest as a model of biological environment.

The present investigation deals with the kinetics of DF oxidation reactions photosensitized by $P_{y}$ in aqueous micellar solutions of SDS and DTAC, and in methanolic solutions, containing $\mathbf{P}_{\mathbf{y}}, \mathrm{DF}$ and sodium azide. $\mathrm{P}_{\mathrm{y}}$ and $\mathrm{DF}$ were controlled to be bound to different micelles in the reaction mixture by sample preparation. From kinetic data obtained, the effect of micelles on the production and reaction of singlet oxygen was discussed. The rate constant for singlet oxygen quenching by empty micelles was determined, and the penetration probability of singlet oxygen from aqueous phase into micellar interior was also estimated. 


\section{Results and Discussion}

Irradiation of the reaction mixture (aqueous micellar dispersion of $0.1 \mathrm{M}$ SDS or $0.1 \mathrm{M}$ DTAC containing solubilized $\mathrm{P}_{\mathbf{y}}$ and $\mathrm{DF}$; saturated with oxygen) with the filtered light $(338 \mathrm{~nm})$ decreased rapidly the concentration of $\mathrm{DF}$; an initial linear decrease with the irradiation time followed by a saturation. $\mathbf{P}_{\mathbf{y}}$ and $\mathrm{DF}$, hydrophobic molecules, in the reaction mixture are presumed to be bound to different micelles separated each other, under the present sample preparation. DF is known to be highly reactive with singlet oxygen and to be oxidized into dibenzoyl benzene [22]. For this reason, the above decrease in $\mathrm{DF}$ concentration is considered to have been caused by the oxidation of DF by the singlet oxygen produced by the photosensitization of $\mathbf{P}_{\mathbf{y}}$, possibly through the triplet energy transfer from $P_{y}$ to oxygen. In the absence of $P_{y}$, the DF concentration was unchanged with a several min-irradiation of the filtered light employed.

When $\mathrm{NaN}_{3}$, a scavenger for singlet oxygen, was added to the reaction mixture, the oxidation of DF was considerably inhibited depending on the $\mathrm{NaN}_{3}$ concentration. $\mathrm{NaN}_{3}$ is watersoluble, and may be distributed in the aqueous phase as the ionized form.

The reaction mixture used in the present investigation contained various micelles; $\mathrm{P}_{\mathbf{y}}$-bound $\left(\mathrm{M}_{\mathbf{P y}}\right)$, DF-bound $\left(\mathbf{M}_{\mathbf{D F}}\right)$ and empty ( $\left.\mathbf{M}_{\text {empty }}\right)$ micelles. The total micelle $\left(\mathbf{M}_{\text {total }}\right)$ concentration was estimated to be $1.6 \cdot 10^{-3}$ and $2.0 \cdot 10^{-3} \mathrm{M}$ for $0.1 \mathrm{M}$ SDS and $0.1 \mathrm{M}$ DTAC, respectively, assuming the aggregation numbers of 62 for SDS micelle [23, 24] and 50 for DTAC micelle [25]. The maximum concentrations of $M_{P y}$ and $M_{D F}$ are equal to those of $P_{y}\left(1 \cdot 10^{-4} M\right)$ and $\mathrm{DF}\left(\leq 6 \cdot 10^{-5} \mathrm{M}\right)$, respectively. Therefore, a large amount of empty micelles was present in the reaction mixture. In order to examine whether empty micelles behave as scavenger for singlet oxygen, the quantum yield $\left(\Phi_{\mathrm{DF}}\right)$ for DF oxidation was measured as a function of DF concentration changing the concentration of empty micelles. The concentration of DF-bound micelles could be neglected compared with that of empty micelles. Results obtained in SDS micellar solutions are shown in Fig. 1. The curves indicate a considerable quenching effect of empty micelles for singlet oxygen. Such a quenching effect was also seen in empty micelles of DTAC. $\Phi_{\mathrm{DF}}$ was determined using the initial velocity of the decrease in DF concentration and the number of photons absorbed by $\mathbf{P}_{\mathbf{y}}$ per min.

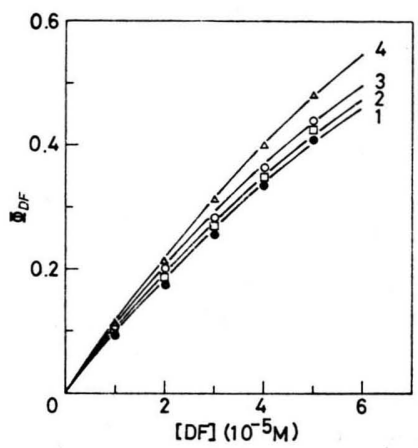

Fig. I. Quantum yield $\left(\Phi_{\mathrm{DF}}\right)$ for DF oxidation as a function of DF concentration at various concentrations of empty SDS micelles. Concentration of $\mathrm{P}_{\mathrm{y}}, \mathrm{I} \cdot 10^{-4} \mathrm{M}$; concentration of empty SDS micelles for Curves 1, 2, 3, and $4,1.5 \cdot 10^{-3}, 1.3 \cdot 10^{-3}, 1.2 \cdot 10^{-3}$ and $0.9 \cdot 10^{-3} \mathrm{M}$, respectively.

Simplified diagram for the photochemical reaction in aqueous micellar solutions containing $\mathrm{P}_{\mathbf{y}}$, $\mathrm{DF}$ and $\mathrm{NaN}_{3}$ is shown in Fig. 2, assuming that $P_{y}$ and DF are bound to the interior of micelles. Then, the reciprocal of the quantum yield for $\mathrm{DF}$ oxidation is represented as follows.

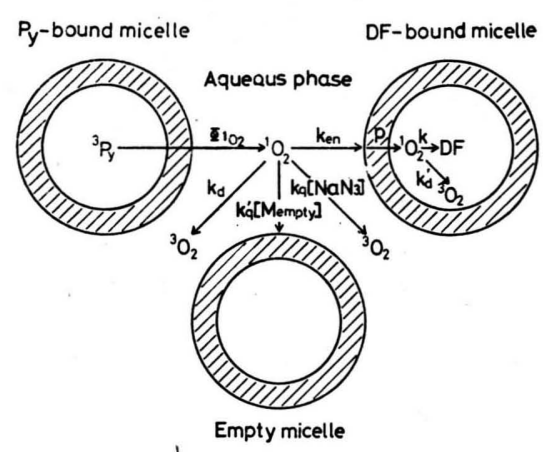

Fig. 2. Simplified diagram for photochemical reaction in aqueous micellar solutions containing $\mathrm{P}_{\mathbf{y}}, \mathrm{DF}$ and $\mathrm{NaN}_{3} . \Phi_{0_{2}}$, quantum yield for singlet oxygen production in aqueous phase; $k_{r}$, overall rate constant for DF oxidation by singlet oxygen produced in aqueous phase; $\mathrm{k}_{\mathrm{q}}$, rate constant for singlet oxygen quenching by $\mathrm{NaN}_{3} ; \mathrm{k}_{\mathrm{q}}$, rate constant for singlet oxygen quenching by empty micelles $\left(\mathbf{M}_{\mathrm{empty}}\right)$; $\mathrm{k}_{\mathrm{d}}$, rate constant for physical decay of singlet oxygen in aqueous phase. $k_{r}=k_{e n} \cdot p \cdot k /\left(k+k_{d}{ }^{\prime}\right)$, where $k_{e n}$, $k$ and $k_{d}^{\prime}$ are rate constants for encounter between singlet oxygen and micelle, for DF oxidation by singlet oxygen in interior of micelle and for physical decay of singlet oxygen in interior of micelle, respectively, and $p$ is penetration probability of singlet oxygen from outside into inside of micelle. ${ }^{3} \mathbf{P}_{\mathbf{y}}$, triplet $\mathbf{P}_{\mathbf{y}}$. 
$\Phi_{\mathrm{DF}}^{-1}=$

$\Phi_{102}^{-1}\left(1+\frac{k_{d}+k_{q}{ }^{\prime}\left[M_{e m p t y}\right]+k_{q}\left[N a N_{3}\right]}{k_{r}} \cdot \frac{1}{[D F]}\right)$

where the overall rate constant for the oxidation reaction of singlet oxygen in aqueous phase with DF bound to micelle,

$$
\mathbf{k}_{\mathbf{r}}=\mathbf{k}_{\mathrm{en}} \cdot \mathbf{p} \cdot \mathbf{k} /\left(\mathbf{k}+\mathbf{k}_{\mathbf{d}}{ }^{\prime}\right) \text {. }
$$

$\Phi_{1_{0}}, \mathrm{k}_{\mathrm{en}}, \mathrm{k}_{\mathrm{q}}, \mathrm{k}_{\mathbf{q}^{\prime}}, \mathrm{k}_{\mathrm{d}}, \mathrm{k}_{\mathrm{d}^{\prime}}, \mathrm{k}$ and $\mathrm{p}$ are illustrated in the legend of Fig. 2. Under the present experimental conditions, the concentration of empty micelles, [ $\left.M_{\text {empty }}\right]$, could be approximated to be nearly equal to that of the total micelles, $\left[\mathrm{M}_{\text {total }}\right]$, because the concentrations of $\mathrm{P}_{\mathbf{y}}$ and $\mathrm{DF}$ were much less than that of the total micelles as mentioned above. From eq. (1), $\Phi_{\mathrm{DF}}^{-1}$ should be proportional to the reciprocal of $\mathrm{DF}$ concentration $\left(\mathrm{k}_{\mathrm{q}}{ }^{\prime}=0\right.$ for methanolic solution). This linear relation was hold for the experimental values obtained in micellar and methanolic solutions as shown in Fig. 3. Plots of $\Phi_{\mathrm{DF}}^{-1}$ in DTAC and methanolic solutions are shown only in the absence of $\mathrm{NaN}_{3}$. The intercepts of the curves with the ordinate give $\Phi_{102}^{-1}$ in micellar and methanolic solutions. Obtained values of $\Phi_{1_{02}}$ are given in Table I. The values of $\Phi_{1_{02}}$ in micellar solutions were higher than that in methanolic solution.

The difference $(\Delta S)$ between slopes for curves in the presence and absence of $\mathrm{NaN}_{3}$ in Fig. 3 is represented as follows,

$$
\Delta \mathrm{S}=\Phi_{102}^{-1} \cdot \mathrm{k}_{\mathrm{q}}\left[\mathrm{NaN}_{3}\right] / \mathrm{k}_{\mathbf{r}} \text {. }
$$

$\Delta \mathrm{S}$ values were determined experimentally at various $\mathrm{NaN}_{3}$ concentrations, and were plotted in Fig. 4 for SDS micellar solutions. The curve obtained

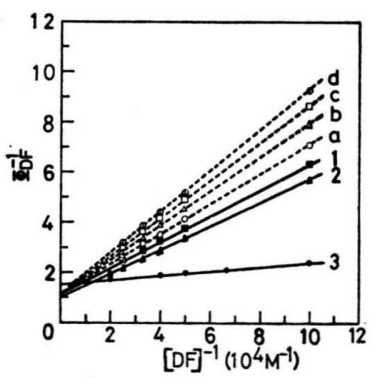

Fig. 3. Plots of reciprocal of quantum yield $\left(\Phi_{D F}\right)$ for DF oxidation against reciprocal of DF concentration. Concentration of $\mathrm{P}_{\mathrm{y}}, 1 \cdot 10^{-4} \mathrm{M}$; Curves 1,2 and 3 in the absence of $\mathrm{NaN}_{3}, 0.1 \mathrm{M}$ SDS, 0.1 M DTAC and methanolic solutions, respectively; $\mathrm{NaN}_{3}$ concentrations for Curves $a, b, c$ and $d$ in 0.1 M SDS solutions, $2 \cdot 10^{-4}, 4 \cdot 10^{-4}, 6 \cdot 10^{-4}$ and $8 \cdot 10^{-4} \mathrm{M}$, respectively.
Table I. Quantum yield $\left.\left(\Phi_{1}\right)_{2}\right)$ for singlet oxygen production, rate constant $\left(k_{r}\right)$ for oxidation reaction of DF by singlet oxygen and rate constant $\left(\mathrm{k}_{\mathrm{q}}\right)$ for singlet oxygen quenching by $\mathrm{NaN}_{3}$ in methanolic and micellar solutions, and rate constant $\left(\mathrm{k}_{\mathrm{q}}{ }^{\prime}\right)$ for singlet oxygen quenching by empty micelles and penetration probability (p) of singlet oxygen from outside into interior of micelle in micellar solutions.

\begin{tabular}{lclccc}
\hline $\begin{array}{l}\text { Solu- } \\
\text { tions }\end{array}$ & $\Phi_{{ }^{2} \mathrm{z}_{\mathrm{z}}}$ & $\begin{array}{c}\mathrm{k}_{\mathrm{r}} \\
{\left[\mathrm{M}^{-1} \mathrm{sec}^{-1}\right]}\end{array}$ & $\begin{array}{c}\mathrm{k}_{\mathrm{q}} \\
{\left[\mathrm{M}^{-1} \mathrm{sec}^{-1}\right]}\end{array}$ & $\begin{array}{c}\mathrm{k}_{\mathrm{q}}{ }^{\prime} \\
{\left[\mathrm{M}^{-1} \mathrm{sec}^{-1}\right]}\end{array}$ & $\mathrm{p}$ \\
\hline SDS & 0.9 & $2.2 \cdot 10^{10}$ & $7.9 \cdot 10^{8}$ & $1.0 \cdot 10^{9}$ & 0.38 \\
DTAC & 1.0 & $2.8 \cdot 10^{10}$ & $1.8 \cdot 10^{9}$ & $1.7 \cdot 10^{8}$ & 0.48 \\
MeOH & 0.6 & $7.8 \cdot 10^{8}$ & $2.3 \cdot 10^{8}$ & - & $(1.0)$ \\
\hline
\end{tabular}

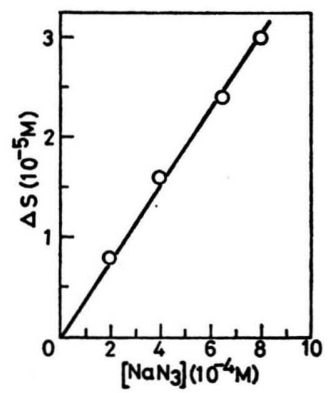

Fig. 4. Difference $(\Delta S)$ between slopes for the curves in the presence and absence of $\mathrm{NaN}_{3}$ in Fig. 3 as a function of $\mathrm{NaN}_{3}$ concentration in $0.1 \mathrm{M}$ SDS solutions. $\Delta \mathrm{S}=\Phi_{1_{2}}-1 \cdot \mathrm{k}_{\mathrm{q}}\left[\mathrm{NaN}_{3}\right] / \mathrm{k}_{\mathrm{r}}$.

was linear again, and its slope gave $\Phi_{102}^{-1} \cdot k_{\mathbf{q}} / k_{\mathbf{r}}$. Next, the slope for the curve in the absence of $\mathrm{NaN}_{3}$ shown in Fig. 3 was determined as a function of $\left[M_{\text {total }}\right]$, and was plotted in Fig. 5 for SDS micellar solutions. The curve obtained was linear with an intercept of $\Phi_{102}^{-1} \cdot k_{d} / k_{r}$ and a slope of $\Phi_{102}{ }^{-1} \cdot \mathbf{k}_{\mathbf{q}}{ }^{\prime} / \mathbf{k}_{\mathbf{r}}$. The same treatment could be made for DTAC micellar solutions. The value of $\Phi_{102}^{-1} \cdot \mathrm{k}_{\mathrm{d}} / \mathrm{k}_{\mathrm{r}}$ for methanolic solution was determined from the slope of the curve in the absence of $\mathrm{NaN}_{3}$ in Fig. 3.

Merkel et al. [12] described that the lifetime $\left(1 / k_{d}\right)$ of singlet oxygen was $2 \mu$ sec in water and

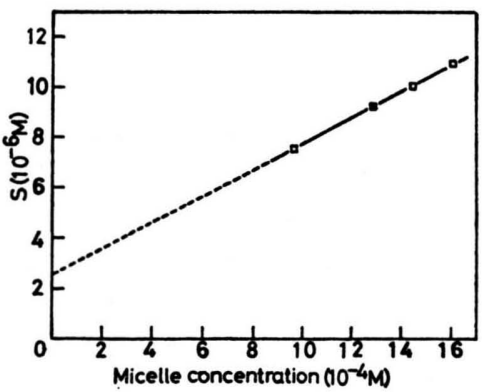

Fig. 5. Slope (S) for the curve in the absence of $\mathrm{NaN}_{3}$ shown in Fig. 3 as a function of SDS micelle concentration. 
$7 \mu$ sec in methanol. Accordingly, assuming that the lifetime of singlet oxygen in aqueous phase of micellar solution is the same as $2 \mu$ sec in water, $\mathbf{k}_{\mathbf{r}}, \mathbf{k}_{\mathbf{q}}$ and $\mathbf{k}_{\mathbf{q}}{ }^{\prime}$ were determined using the obtained values of $\Phi_{102}^{-1} \cdot k_{q} / k_{r}, \Phi_{102}^{-1} \cdot k_{d} / k_{r}$ and $\Phi_{102}^{-1} \cdot k_{q}{ }^{\prime} / k_{r}$, as given in Table $I$. The magnitude of $k_{r}$ values was in the order of DTAC solution $>$ SDS solution $>$ methanolic solution. Using methylene blue as sensitizer, Merkel et al. [12] obtained $\mathrm{k}_{\mathrm{r}}=8$. $10^{8} \mathrm{M}^{-1} \mathrm{sec}^{-1}$ in methanolic solution, and Young et al. [8] obtained $\mathrm{k}_{\mathrm{r}}=1.3 \cdot 10^{9} \mathrm{M}^{-1} \mathrm{sec}^{-1}$ in methanolic solution and $\mathrm{k}_{\mathrm{r}}=1.2 \cdot 10^{9} \mathrm{M}^{-1} \mathrm{sec}^{-1}$ in dioxanic solution. Gorman et al. [21] estimated $\mathrm{k}_{\mathrm{d}} / \mathrm{k}_{\mathrm{r}}=9.7 \cdot 10^{-5} \mathrm{M}$ in SDS micellar solutions with methylene blue as sensitizer, which is higher than our $k_{d} / k_{r}$ value of $2.3 \cdot 10^{-5} M$. The high $k_{d} / k_{r}$ value obtained by them may be due to neglect of singlet oxygen quenching by empty micelles. The magnitude of $k_{q}$ values was also in the above order. Hasty et al. [17] obtained $\mathrm{k}_{\mathrm{q}}=2.2 \cdot 10^{8} \mathrm{M}^{-1} \mathrm{sec}^{-1}$ in methanolic solution using methylene blue as sensitizer. A larger $\mathbf{k}_{\mathbf{q}}$ value in DTAC micellar solution than that in SDS micellar solution might be derived from the difference in distribution of azide ions due to counter-ion effect. DTAC micelles are cationic and SDS micelles anionic. The rate constants for singlet oxygen quenching by empty micelles were surprisingly large comparable to those by $\mathrm{NaN}_{3}$, especially in SDS micellar solution.

Gorman et al. [21] calculated the rate constant for encounter between excited oxygen and SDS micelles from the expression of Weston and Schwarz $[26], \mathrm{k}_{\mathrm{en}}=4 \pi \mathrm{a}\left(\mathrm{D}_{\Delta}+\mathrm{D}_{\mathrm{m}}\right)$, assuming that the diffusion coefficients of oxygen $\left(\mathrm{D}_{\Delta}\right)$ [26] and SDS micelles $\left(D_{m}\right)$ [27] in water are $5 \cdot 10^{-9}$ and $1 \cdot 10^{-10}$ $\mathrm{m}^{2} \mathrm{sec}^{-1}$, respectively, and the collision radius of the encounter pair (a) is nearly equal to the micellar radius $\left(1.5 \cdot 10^{-9} \mathrm{~m}\right)$. They obtained $\mathrm{k}_{\mathrm{en}}=5.8 \cdot 10^{10}$ $\mathrm{M}^{-1} \mathrm{sec}^{-1}$. We estimated the penetration probability (p) of singlet oxygen from aqueous phase into the interior of micelle from eq. (2) with the assumption of $\mathbf{k} \gg \mathbf{k}_{\mathbf{d}^{\prime}}$ in the interior of micelle and $\mathbf{k}_{\mathrm{en}}=$ $5.8 \cdot 10^{10} \mathrm{M}^{-1} \mathrm{sec}^{-1}$ for SDS and DTAC micellar solutions, using the $k_{r}$ values obtained. The lifetime of singlet oxygen seems to be larger in nonpolar solvents than that in polar solvents [12]. Calculated $p$ values are given in Table I. Of course, p value for methanolic solution without micelles corresponds to unity. From $p$ values obtained, singlet oxygen in aqueous phase seems to penetrate into the interior of DTAC micelle more efficiently than into that of SDS micelle.

The quantum yield for singlet oxygen production sensitized by methylene blue has been estimated to be 0.52 in methanolic solution [28]. This value is comparable to the quantum yield obtained with sensitizer $\mathbf{P}_{\mathbf{y}}$ in methanolic solution. The quantum yield for singlet oxygen production in micellar solutions has not been obtained so far as we know. $\mathbf{P}_{\mathbf{y}}$, a hydrophobic molecule, was a good photosensitizer for singlet oxygen production in SDS and DTAC micellar solutions, and the quantum yield $\left(\Phi_{1_{02}}\right)$ was very close to unity. Gorman et al. [21] estimated the penetration probability of $\sim 0.1$ for SDS micellar solutions, using $\mathrm{k}_{\mathrm{r}}=5.2 \cdot 10^{9} \mathrm{M}^{-1}$ $\sec ^{-1}$. ( $k_{d}=5 \cdot 10^{5} \mathrm{sec}^{-1}$ was assumed.) The quantum yield $\left(\Phi_{10_{2}}\right)$ in the present investigation was defined as the yield of singlet oxygen produced in aqueous phase (not in micellar interior). Next, it is notable that $\mathbf{k}_{\mathbf{q}}$ ' values were large comparable to $k_{\mathbf{q}}$ values. Empty micelles seem to act as efficient scavenger for singlet oxygen. $P_{y}$-bound micelles may also trap singlet oxygen. In this case, the singlet oxygen trapped may be regenerated by the photosensitization of $P_{y}$ even if it is quenched to the ground state in the inerior of micelle.

In conclusion, $P_{y}$ bound to micelles effectively photosensitized the oxidation of $\mathrm{DF}$ bound to micelles with large rate constants and high quantum yields for singlet oxygen production. At present, the detailed mechanism for these can not be given merely from the results obtained. The micellar structure, the distribution of molecular oxygen, the production site of singlet oxygen, the lifetime of singlet oxygen in aqueous phase and interior of micelle, and the binding site of $P_{y}$ and DF are still open to be elucidated. To clarify these points may lead to the full understanding of the mechanism of photochemical reactions caused in micellar solutions.

\section{Materials and Methods}

SDS and DTAC were obtained from Nakarai Chemical Co. and Maruwaka Kagaku Co., respectively, and were used without further purification. $\mathrm{P}_{\mathrm{y}}, \mathrm{NaN}_{3}$ and $\mathrm{MeOH}$ purchased from Katayama Chemical Co. were guaranteed reagents. DF was of special grade for analytical use and was obtained from Aldrich Chemical Co. Laboratory-distilled water was further distilled from alkaline $\mathrm{KMnO}_{4}$ solution. 
Crystalline $\mathbf{P}_{\mathbf{y}}$ or $\mathrm{DF}$ was dissolved by stirring the crystals in warm $0.1 \mathrm{M}$ aqueous solution $\left(60^{\circ} \mathrm{C}\right)$ of SDS or DTAC in the dark. Sufficient dissolution was obtained in 4-7 h. The concentrations of $P_{y}$ and $\mathrm{DF}$ in the original micellar solutions were $1 \cdot 10^{-3}$ and $1 \cdot 10^{-4} \mathrm{M}$, respectively. The reaction mixture was prepared by mixing the original micellar solutions containing $\mathbf{P}_{\mathbf{y}}$ and $\mathrm{DF}$ separately before irradiation. When necessary, the mixed solution was diluted with $\mathrm{P}_{\mathrm{y}}$ and $\mathrm{DF}$ free micellar solution or water. The concentrations of $P_{y}$ and DF were determined by absorbances at 338 and $415 \mathrm{~nm}$, respectively.

The reaction mixture in a quartz vessel $\left(1 \times 1 \times 4 \mathrm{~cm}^{3}\right)$ was irradiated with the ultraviolet light isolated from a $150 \mathrm{~W}$ xenon lamp through an interference filter (peak, $338 \mathrm{~nm}$; half width, $22 \mathrm{~nm}$; Nippon Shinku Kogaku type S) at $40{ }^{\circ} \mathrm{C}$. Fig. 6 shows the absorption spectra of solubilized $\mathrm{P}_{\mathrm{y}}$ and $\mathrm{DF}$ in aqueous micellar solution of 0.1 M SDS and the transmission of the interference filter. The concentrations of $\mathrm{P}_{\mathrm{y}}$ and $\mathrm{DF}$ employed in the present investigation were $1 \cdot 10^{-4}$ and $5 \cdot 10^{-6}-6 \cdot 10^{-5} \mathrm{M}$, respectively. Therefore, in the reaction mixtures (SDS, DTAC and $\mathrm{MeOH}$ solutions containing $\mathbf{P}_{\mathrm{y}}$ and $\mathrm{DF}$ ) used, the amount of photons absorbed directly by DF was estimated to be less than $2 \%$ of the photons absorbed by $\mathrm{P}_{\mathbf{y}}$. $\mathrm{DF}$ is oxidizable with direct excitation, but the present experimental conditions could neglect this kind of DF oxidation. The amount of photooxidized DF was determined by the decrease in absorbance at $415 \mathrm{~nm}$. The number of photons absorbed by $P_{y}$ was determined by the potassium ferrioxalate method (sensitive to $334 \mathrm{~nm}$ light) of Hatchard and
Parker [29]. The actinometer cell containing 1.25 $\mathrm{mM}$ potassium ferrioxalate in $0.1 \mathrm{~N} \mathrm{H}_{2} \mathrm{SO}_{4}$ was placed just behind the target cell. Under the present experimental conditions, $1 \cdot 10^{-4} \mathrm{M} \mathrm{P}_{\mathrm{y}}$ in $\mathrm{MeOH}$ and micellar solutions of SDS and DTAC absorbed $3.8 \cdot 10^{18}, 3.3 \cdot 10^{18}$ and $3.6 \cdot 10^{18}$ photons per min at the target position, respectively.

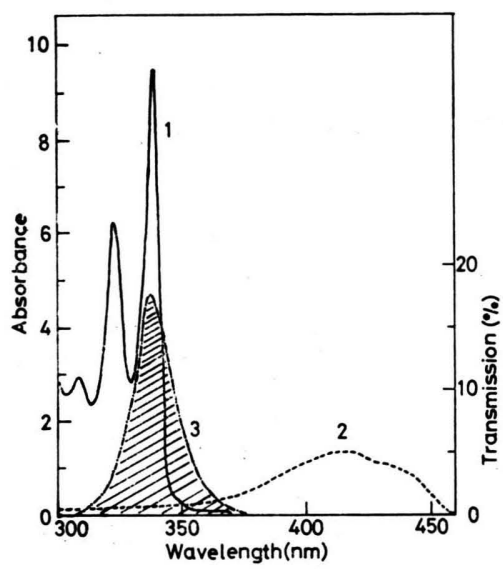

Fig. 6. Absorption spectra of $\mathrm{P}_{\mathrm{y}}$ and $\mathrm{DF}$ in SDS micellar solutions, and transmission of the interference filter used. Concentrations of SDS, $\mathrm{P}_{\mathrm{y}}$ and DF, 0.I, $1 \cdot 10^{-4}$ and $1 \cdot 10^{-4} \mathrm{M}$, respectively; Curve $1, P_{\mathbf{y}}$; Curve 2, DF; Curve 3, transmission.

Absorption spectra were measured with a Hitachi spectrophotometer type 124, and the fluorescence spectra with a Hitachi spectrofluorimeter type 203 modifying the irradiating and recording parts.
[I] E. A. Ogryzlo, in A. C. Giese (ed.): Photophysiology Vol. V, pp. 35-47, Academic Press, New York and London 1970.

[2] T. Wilson and J. W. Hastings, in A. C. Giese (ed.): Photophysiology Vol. V, pp. 49-95, Academic Press, New York and London 1970.

[3] P. B. Merkel and D. R. Kearns, Chem. Phys. Lett. 12, 120 (1971).

[4] D. R. Kearns, Chem. Rev. 71, 395 (I97I).

[5] C. S. Foote, Science (Washington) 162, 963 (1968).

[6] D. F. Evans and J. N. Tucker, J. Chem. Soc. Faraday Trans. II, 72, 166I (1976).

[7] R. H. Young, K. Wehrly, and R. L. Martin, J. Am. Chem. Soc. 93, 5774 (I97I).

[8] R. H. Young, D. Brewer, and R. A. Keller, J. Am. Chem. Soc. 95, 375 (1973).

[9] I. Saito, K. Inoue, and T. Matuura, Photochem. Photobiol. 21, 27 (1975).

[10] Y. Usui, Chemistry Lett. (Japan) 1973, 743.

[II] C. S. Foote and R. W. Denny, J. Am. Chem. Soc. 90,6233 (1968).

[12] P. B. Merkel and D. W. Kearns, J. Am. Chem. Soc. 94, 7244 (1972).
[13] C. A. Long and D. Kearns, J. Am. Chem. Soc. 97, 2018 (1975)

[14] Y. Usui and K. Kamogawa, Photochem. Photobiol. 19, 245 (1974).

[15] D. R. Adams and F. Wilkinson, J. Chem. Soc. Faraday Trans. II, 68, 586 (I972).

[16] R. Nilsson and D. R. Kearns, Photochem. Photobiol. 19, I8I (1974).

[17] N. Hasty, P. B. Merkel, P. Radlick, and D. R. Kearns, Tetrahedron Lett. 1972, 49.

[18] H. Berg, F. A. Gollmick, H. E. Jacob, and H. Triesel, Photochem. Photobiol. 16, I25 (1973).

[19] I. B. C. Matheson and J. Lee, J. Am. Chem. Soc. 94, 3310 (1972).

[20] B. M. Monroe, J. Phys. Chem. 81, 1861 (I977).

[21] A. A. Gorman, G. Lovering, and M. A. Rodgers, Photochem. Photobiol. 23, 399 (I976).

[22] C. Dufraisse and S. Ecary, C. R. Acad. Sci. (Paris) 223, 735 (1946).

[23] K. J. Mysels and J. H. Prince, J. Phys. Chem. 63, 1696 (1959).

[24] E. J. Fendler and J. H. Fendler, Adv. Phys. Org. Chem. 8, 271 (1970). 
[25] I. Tabuse, Yukagaku (Oil Chemistry) (Tokyo) 22, I3 (1973) (in Japanese).

[26] R. E. Weston and H. A. Schwartz, In Chemical Kinetics, pp. 160-164, Prentice-Hall, New York 1972.

[27] D. Stigtel, R. J. Williams, and K. J. Mysels, J. Phys. Chem. 59, 330 (I955).
[28] M. Nemoto, H. Kokubun, and M. Koizumi, Bull. Chem. Soc. (Japan) 42, 2464 (1964).

[29] C. G. Hatchard and C. A. Parker, Proc. Roy. Soc. (London) A 235, 518 (1956). 